\title{
Correction to: Helicobacter-induced gastric inflammation alters the properties of gastric tissue stem/progenitor cells
}

Wataru Shibata ${ }^{1,2}$, Soichiro Sue ${ }^{1}$, Sachiko Tsumura ${ }^{1,3}$, Yasuaki Ishii ${ }^{1}$, Takeshi Sato ${ }^{1}$, Eri Kameta ${ }^{1}$, Makoto Sugimori ${ }^{1}$, Hiroaki Yamada ${ }^{1}$, Hiroaki Kaneko', Tomohiko Sasaki ${ }^{1}$, Tomohiro Ishii ${ }^{1}$, Toshihide Tamura', Masaaki Kondo ${ }^{1}$ and Shin Maeda ${ }^{1 *}$

\section{Correction}

Unfortunately, the original article [1] contained an error incorporated during production. A duplicated version of Table 1 was published in place of Table 2. Table 2 has been corrected in the original article and is also included correctly below.

Table 2 List of representative genes differentially expressed between organoids with and without $H$. felis infection

\begin{tabular}{ll}
\hline Gene Name & Log2 ratio \\
\hline Intestine related genes & 2.75 \\
Isx & 2.10 \\
Muc4 & 1.70 \\
Muc16 & 1.10 \\
Vil1 & \\
Gastric genes & -3.17 \\
Pepsinogen C & -2.47 \\
Gif & \\
Others & 3.30 \\
Gastrin & 2.77 \\
Cd34 & 2.51 \\
II-1a & 1.31 \\
Cd274 (Pd-11) & \\
\hline
\end{tabular}

\author{
Author details \\ 'Department of Gastroenterology, Yokohama City University Graduate School \\ of Medicine, Yokohama, Japan. ${ }^{2}$ Division of Translational Research, Advanced \\ Medical Research Center, Yokohama City University, Yokohama, Japan. \\ ${ }^{3}$ School of Medicine, Yokohama City University, Yokohama, Japan.
}

Published online: 08 January 2018

\section{Reference}

1. Shibata W, et al. Helicobacter-induced gastric inflammation alters the properties of gastric tissue stem/progenitor cells. BMC Gastroenterol. 2017; 17:145. https://doi.org/10.1186/s12876-017-0706-6.

*Correspondence: smaeda@med.yokohama-cu.ac.jp

'Department of Gastroenterology, Yokohama City University Graduate School

of Medicine, Yokohama, Japan 